\title{
Determination and comparison of time under tension required to perform 8, 10 and 12-RM loads in the bench press exercise
}

\author{
Jurandir Baptista da Silva ${ }^{1,2}$, Vicente Pinheiro Lima1,2,3, Gabriel Andrade Paz ${ }^{2,3}$, Caroline Reis \\ de Oliveira ${ }^{2}$, Francisco D'urso ${ }^{2}$, Rodolfo de Alkmim Moreira Nunes ${ }^{1}$, Juliana Brandão Pinto \\ de Castro $^{1}$, Rodrigo Gomes de Souza Vale ${ }^{1}$ \\ 1 Institute of Physical Education and Sports, Postgraduate Program in Exercise and Sport Sciences, State University of \\ Rio de Janeiro, Rio de Janeiro, Brazil; ${ }^{2}$ Biodynamic Laboratory of Exercise, Health and Performance, Castelo Branco \\ University, Rio de Janeiro, Brazil; ${ }^{3}$ Biodesp Institute, Kinesiology Center of Performance, Rio de Janeiro, RJ, Brazil
}

\section{Summary}

Study aim: To determine and compare the time under tension (TUT) required to perform 8, 10 and 12 repetition maximum (RM) loads in the bench press exercise.

Material and methods: Twenty men $(24.17 \pm 4.69$ years $)$ were selected intentionally and properly. We included in the study physically active individuals, with a weekly frequency of physical activity of at least two days for six months, and excluded individuals with injury or pain that could interfere with the correct execution of the exercise and individuals with positive PAR-Q. The 10-RM test consisted in performing ten consecutive repetitions with maximum overload and the highest speed in bench press exercise on the Smith machine. After 48 h, 10-RM sets were performed with the load obtained in the 10-RM load testing. The TUT in 10-RM was verified through kinematics using the timing technique of the Kinovea software.

Results: The RM loads and TUT obtained during the retest session showed normal distribution between subjects. However, no significant differences were found between the loads 8, 10 and 12-RM within and between subjects $(\mathrm{p}<0.05)$. The verified TUT showed a difference from 8 to $10-\mathrm{RM}$ and from 8 to 12-RM, but no significant difference was found between TUT protocols for 10 and $12-\mathrm{RM}(\mathrm{p}<0.05)$.

Conclusion: The study results enable evaluation of TUT in bench press exercise on the Smith machine for the study sample, allowing, for this group, the prediction and control of training intensity through the TUT.

Keywords: Resistance training - Time under tension - 10-RM - Bench press

\section{Introduction}

Resistance training (RT) has been prescribed for individuals who want to optimize muscle hypertrophy and strength gains [12, 22, 29]. For such results, some variables must be controlled and manipulated, such as increased overload, number of repetitions, sets, frequency, exercise order, and rest interval between sets [16]. The overload and the number of repetitions determine the intensity of the training. The intensity can be determined by the zone of repetition maximum (RM), which is a relevant variable to be observed $[1,11]$. The training intensity is also prescribed using the percentages of 1-RM $[8,9,25]$.
However, there is some time interval during the RM execution. Thus, the intensity can also be characterized by the total time of tension under a period of time [17]. Therefore, in the implementation of one or more training $\mathrm{RM}$, there is a certain interval of time covered by the production of muscle strength, which is the time under tension (TUT). This is proportional to the number of goal repetitions because muscle tension is related to the product of force and displacement.

Fleck and Kraemer [11] recommend training using 8 to 12 -RM loads with the goal of increasing the hypertrophy gains. However, the magnitude of the hypertrophic response depends not only on exercise intensity, but also on the time the muscle is under tension [7]. The execution of strength exercises at different speeds changes 
the TUT and consequently the results [19]. Burd et al. [7] verified the muscular activation and protein synthesis in carrying out the exercise extensor chair, with the intensity controlled to $30 \%$ of $1-\mathrm{RM}$, at two different speeds of execution, thus changing the final TUT. The results showed that different times under tension significantly alter the muscular response. However, Lacerda et al. [17] found that the same TUT can also present different results in the electromyographic activity carried out at different running speeds and consequently different number of repetitions. Thus, these studies point to the need to determine the average TUT of exercise and its influence on muscle responses.

The duration of the repetition influences both the training load [26] and the acute and chronic adaptations arising from a strength-training program [30]. Some studies have indicated that the duration of repetition can influence the maximum strength performance [23] and muscular power [5]. For training aiming at muscular hypertrophy, generally moderate to high speeds are suggested, depending on the individual's training condition $[1,16]$. Some authors suggest values around 5 seconds [4], while others suggest a range of 2 to 6 seconds [33]. Repetition durations of 4 and 6 seconds are traditionally recommended [24]. However, there is no clear justification for such recommendations, because the average TUT for exercises is not well described in the literature.

Thus, there is a need for further studies to examine the muscular responses in the control of strength training variables, in particular, the prescription of exercise intensity from the time under tension $[13,15,32]$. However, there is still a lack of evidence about the TUT required to perform 8,10 and 12-RM testing. In this context, it is believed that there will be a decrease in the load used for the largest number of repetitions (8 versus 12) and consequently in strength. However, it allows the achievement of higher speeds in the initial phase, which may allow the assimilation of TUT in these executions.

Therefore, the purpose of this study was to determine and compare the time under tension required to perform 8 , 10 and 12-RM loads in the bench press exercise.

\section{Material and methods}

\section{Participants}

This is a descriptive and comparative study [31]. Male military personnel of Rio de Janeiro, Brazil, were invited to join this study. Twenty physically active individuals were selected by convenience to participate in the intervention. To be included in the study, a subject had to have a physical activity frequency of at least two days a week and practice physical exercise regularly for at least six months. Individuals with injury or pain that could interfere with the correct execution of the exercise and those with a positive PAR-Q [28] were excluded from the study.

The participants who agreed to participate in the study signed an informed consent form in accordance with the guidelines regarding human research delineated in Resolution 466/2012 of the National Health Council [10] and the Declaration of Helsinki [35].

The description of the sample includes measurements of body mass and stature (Table 1). The measurement of body mass (expressed in kilograms) was performed on a mechanical scale (Filizola, Brazil) and stature (expressed in meters) using a portable stadiometer (Seca, Baystate Scale \& Systems, USA). Additionally, we calculated the body mass index (BMI) [20] as the ratio between the body mass and the square of the stature $\left(\mathrm{kg} / \mathrm{m}^{2}\right)$. We adopted the three skinfolds protocol proposed by Jackson and Pollock [14] to estimate the percentage [\%] of body fat.

\section{Eight, ten and twelve-repetition maximum load determination}

The repetition maximum load test consists of performing consecutive repetitions with maximum overload at the highest possible pace [2]. The test was stopped when the movement was performed with the incorrect technique and/or when there occurred voluntary concentric failures at 8,10 and 12-RM. In order to reduce the margin of error in the tests, the following strategies were adopted: a) standardized instructions were supplied before the test, so that the appraised was aware of the whole routine that

Table 1. Characteristics of the sample

\begin{tabular}{lccccc}
\hline & Age [years] & Body mass $[\mathrm{kg}]$ & Stature [m] & BMI & Body fat [\%] \\
\hline Mean & 24.17 & 80.85 & 1.72 & 27.14 & 11.24 \\
SD & 4.69 & 14.86 & 0.06 & 4.20 & 4.50 \\
Minimum & 20.00 & 58.60 & 1.58 & 22.06 & 6.70 \\
Maximum & 35.00 & 117.00 & 1.80 & 36.93 & 23.60 \\
\hline
\end{tabular}


involved the data collection; b) the appraised was instructed on the execution technique of the exercise; c) the examiner was aware regarding the position adopted by the test of time, because small variations in the positioning of the joints involved in the movement could work other muscles, leading to erroneous interpretations of the obtained scores; d) verbal stimulus was provided in order to maintain a high level of motivation. The intervals between attempts during the tests were fixed at five minutes. The intervals between protocols were 48 hours. The subjects were not to consume any stimulant drink (caffeine or alcohol) or perform physical activity 48 hours before the tests.

The position of the individual in bench press in the apparatus Smith Machine (TechnoGym, Rio de Janeiro, Brazil) was with both feet on the floor, column with physiological curvatures preserved, shoulder in abduction of $90^{\circ}$ and elbows flexed at $90^{\circ}$. In this position, the back of the arm was touching a rope sustained by two trestles limiting the lower amplitude. In the execution of the exercise, horizontal adduction of shoulder, abduction of shoulder girdle and full elbow extension to $0^{\circ}$ were carried out. To verify the time of beginning and ending of the movement, as well as the behavior of the angular and linear joint kinematics, we attached reflective markers on the wrists, elbows and shoulders of the participants, ensuring the movement pattern. The images were acquired by a camera (Sony, Japan) positioned on a tripod in order to allow the full view of the movement and were analyzed with Kinovea software. The failure of the movement, as well as the withdrawal of the seat back and/or legs off the ground, was observed. If the execution was not in accordance with the standards, the collection was canceled and rescheduled. The TUT measurements were assessed through the kinematics using the timing technique of the Kinovea software [3].

\section{Statistical analysis}

Data were analyzed by SPSS 20 for Windows and presented as maximum, minimum, mean and standard deviation. Normality and variance homogeneity of data were determined using Shapiro-Wilk and Levene tests, respectively. One-way ANOVA was applied for comparisons between RM and TUT protocols, followed by the Bonferroni post-hoc test to identify possible differences. The significance level was set at $\mathrm{p}<0.05$ for all tests.

\section{Results}

Table 2 shows the mean, standard deviation, minimum and maximum of 8,10 and 12-RM load determination and the TUT of the sample.

\section{Discussion}

The main findings of the current study suggested that the RM loads and TUT obtained during the retest session showed normal distribution between subjects. However, no significant differences were found between the loads 8,10 and $12-\mathrm{RM}$ within and between subjects $(\mathrm{p}<0.05)$. The verified TUT presented a significant difference from 8 to $10-\mathrm{RM}$ and from 8 to $12-\mathrm{RM}$. However, no significant difference was found between TUT protocols for 10 and 12-RM ( $<<0.05)$.

Different execution speeds can lead to changes in the measurement of intensity and results. Keeler et al. [15] observed an increase in strength of 8 and 12 repetitions performed with the total time of tension about 48 and $72 \mathrm{~s}$, respectively, and $2 \mathrm{~s}$ in the concentric phase (CP) and $4 \mathrm{~s}$ in the eccentric phase (EP) compared to the super slow speed, with the total tension time of 120 and $160 \mathrm{~s}$, respectively, $10 \mathrm{~s}$ and $5 \mathrm{~s}$ in CP in EP in sedentary women in the bench press. These results are in contrast to the findings of this study, which found a lower TUT for physically active men. However, the present study protocol was based on the TUT for 10-RM and according to ACSM [1]. This number of repetitions is indicated to promote gains of strength and hypertrophy. The different TUT results are explained by the fact that the protocols have been made at different speeds: in the study of Keeler et al. [15] at controlled speeds, and at full speed in this sample. Strength

Table 2. Values of load determination and time under tension for the repetitions

\begin{tabular}{lcccccc}
\hline & \multicolumn{2}{c}{ 8-RM } & \multicolumn{2}{c}{ 10-RM } & \multicolumn{2}{c}{ 12-RM } \\
\hline LD & TUT & LD & TUT & LD & TUT \\
\hline SD & 78.33 & 16.31 & 73.67 & $18.91^{*}$ & 69.33 & $21.80^{+}$ \\
Minimum & 10.16 & 1.06 & 11.15 & 2.69 & 11.13 & 3.16 \\
Maximum & 64.00 & 14.38 & 56.00 & 14.61 & 50.00 & 17.81 \\
\hline
\end{tabular}

SD - Standard deviation; LD - Load determination; TUT - time under tension; ${ }^{*}$ difference from 8 to 10 -RM; ${ }^{+}$difference from 8 to 12 -RM. 
gains and hypertrophy can also be explained by the fact that Keeler et al. [15] used sedentary women in their sample, whereas this protocol used physically active men.

Santiago et al. [27] verified the TUT in leg press exercise and found a TUT for 10-RM of $25.7 \pm 6 \mathrm{~s}$ in trained women. This result was much higher than the TUT values found in this study using the bench press exercise in active men. This suggests that the TUT can vary for the same number of repetitions in exercises, genders and different fitness levels. Thus, this result emphasizes the need to check the TUT for each exercise for each muscle group, and therefore the body segment size can influence the displacement and therefore the speed and TUT in the execution of the exercise.

Gentil et al. [13] compared four different strength training methods using the same overload in the exercise leg extension. In the method with the traditional protocol of 10-RM, the speed of $2 \mathrm{~s}$ for the concentric phase and $2 \mathrm{~s}$ for the eccentric, there was a TUT total of $42.08 \pm 3.18 \mathrm{~s}$. This differs from the present study that found a much lower TUT. These results can be justified because the execution speed was different between studies, which is as fast as possible and held in multi-joint exercise for the upper limbs. Gentil et al. [13] also found that the speed of different execution protocols changes the number of repetitions performed, thus interfering directly with the TUT, holding the number of repetitions and the results, as there were found higher lactate levels in a smaller number of repetitions, but a higher TUT was generated compared to the traditional protocol. These results also suggest that the same number of repetitions may generate different TUT for different exercises and muscle groups.

Lamas et al. [18] evaluated the responses of strength training compared to power training. The strength training group (STG) performed squats with loads between 60 and $95 \%$ of 1-RM, while the power training group (PTG) performed squats with loads between 30 and $60 \%$ of $1-\mathrm{RM}$. Both protocols were performed at the highest possible speed. Although not checking the TUT directly, when considering that the intensity of the load employed in the PTG allows higher speeds to be achieved, this generated a lower TUT compared to STG. However, there was no significant difference in the increase of maximum strength and hypertrophy between protocols. These results suggest that the degree of muscle tension acting at the time of breaking is high enough to the point of stimulating muscle hypertrophy in power training. Thus, this result leads us to believe that the speed of these two protocols may have been the reason for similar results. Although the present study was based on absolute values of RM, such results support the proposal of this study, suggesting the prediction and control of the intensity by the maximum speed and timing.
While not diminishing the importance of the present research, there are some limitations in this study that should be mentioned: first, this study did not take into account the previous practice of the proposed exercise. Additionally, because of the small sample size, the obtained results should be interpreted and generalized with caution.

Summing up, it was possible to achieve the proposed objective of this research, checking the TUT in bench press exercise on the Smith machine for the study sample, allowing, for this group, the prediction and control of training intensity through the TUT. The study hypothesis was partially confirmed, since no differences were observed between the TUT from 10 to 12 -RM. It is recommended that further studies on TUT be performed with both genders, trained and untrained individuals, other exercises and other RM numbers. We also recommend the use of other techniques to analyze responses of TUT such as electromyography and biochemical markers.

\section{References}

1. American College of Sports Medicine (2009) American College of Sports Medicine position stand. Progression models in resistance training for healthy adults. Med. Sci. Sports Exerc., 41: 687-708. DOI: 10.1249/ MSS.0b013e3181915670

2. American College of Sports Medicine (2009) ACSM's guidelines for exercise testing and prescription. 8th ed. Philadelphia: The Point.

3. Balsalobre-Fernández C., C.M. Tejero-González, J. Campo-Vecino, N. Bavaresco (2014) The concurrent validity and reliability of a low-cost, high-speed camera-based method for measuring the flight time of vertical jumps. J. Strength Cond. Res., 28: 528-533. DOI: 10.1519/ JSC.0b013e318299a52e

4. Bird S.P., K.M. Tarpenning, F.E. Marino (2005) Designing resistance training programmes to enhance muscular fitness: a review of the acute programme variables. Sports Med., 35: 841-851.

5. Bottaro, M., S.N. Machado, W. Nogueira, R. Scales, J. Veloso (2007) Effect of high versus low-velocity resistance training on muscular fitness and functional performance in older men. Eur. J. Appl. Physiol., 99: 257-264. DOI: 10.1007/s00421-006-0343-1

6. Brentano M.A., E.L. Cadore, E.M. Silva, R.F. Silva, L.F.M. Kruel (2008) Maximal strength estimation in resistance training exercises based on anthropometric parameters of physically active men and women. Braz. J. Biomotricity, 2: 294-301.

7. Burd N.A., R.J. Andrews, D.W. West, J.P. Little, A.J. Cochran, A.J. Hector, J.G.A. Cashaback, M.J. Gibala, R. Potvin, S.K. Baker, S.M. Phillips (2012) Muscle time under tension during resistance exercise stimulates 
differential muscle protein subfractional synthetic responses in men. J. Physiol., 590: 351-362. DOI: 10.1113/ jphysiol.2011.221200

8. Candow D.G., D.G. Burke (2007) Effect of short-term equal-volume resistance training with different workout frequency on muscle mass and strength in untrained men and women. J. Strength Cond. Res., 21: 204-207. DOI: 10.1519/R-19785.1

9. Chestnut J.L., D. Docherty (1999) The effects of 4 and 10 repetition maximum weight-training protocols on neuromuscular adaptations in untrained men. J. Strength Cond. Res., 13: 353-359.

10. Conselho Nacional de Saúde (Brasil). Resolução no 466, de 12 de dezembro de 2012. Diário Oficial da União, Brasília, DF, n. 12, p. 59, jun. 2013.

11. Fleck S.J., W.J. Kraemer (2014) Designing resistance training programs. 4th ed. Champaign, IL: Human Kinetics.

12. Garber C.E., B. Blissmer, M.R. Deschenes, B.A. Franklin, M.J. Lamonte, I.M. Lee, D.C. Nieman, D.P. Swain (2011) American College of Sports Medicine position stand. Quantity and quality of exercise for developing and maintaining cardiorespiratory, musculoskeletal, and neuromotor fitness in apparently healthy adults: guidance for prescribing exercise. Med. Sci. Sports Exerc., 43: 1334-1359. DOI: 10.1249/MSS.0b013e318213fefb

13. Gentil P., E. Oliveira, M. Bottaro (2006) Time under tension and blood lactate response during four different resistance training methods. J. Physiol. Anthropol., 25: 339-344.

14. Jackson A.S., M.L. Pollock (1978) Generalized equations for predicting body density of men. Br. J. Nutr., 40: 497-504.

15. Keeler L.K., L.H. Finkelstein, W. Miller, B. Fernhall (2001) Early-phase adaptations of traditional-speed vs superslow resistance training on strength and aerobic capacity in sedentary individuals. J. Strength Cond. Res., 15: 309-314.

16. Kraemer W.J., N.A. Ratamess (2004) Fundamentals of resistance training: progression and exercise prescription. Med. Sci. Sports Exerc., 36: 674-688.

17. Lacerda L.T., H.C. Martins-Costa, R.C. Diniz, F.V. Lima, A.G. Andrade, F.D. Tourino, M.G. Bemben, M.H. Chagas (2016) Variations in repetition duration and repetition numbers influence muscular activation and blood lactate response in protocols equalized by time under tension. J. Strength Cond. Res., 30: 251-258. DOI: 10.1519/ JSC.0000000000001044

18. Lamas L., C. Ugrinowitsch, G.E.R. Campos, M.S. Aoki, R. Fonseca, M. Regazzini, A.S. Moriscot, V. Tricoli (2007) Strength training x power training: performance changes and morphological adaptations. Rev. Bras. Educ. Fís. Esp., 21: 331-340.

19. MacDougall J.D. (1986) Adaptability of muscle to strength training: a cellular approach. In: B. Saltin (ed.)
Biochemistry of exercise VI. Champaign, IL: Human Kinetics. pp. 501-513.

20. Marfell-Jones M., T. Olds, A. Stewart, L. Carter (2006) International standards for anthropometric assessment. Potchefstroom, South Africa: ISAK.

21. Marques G.C.O., M.A. Brentano, L.F.M. Kruel (2009) Estimativas da força máxima dinâmica através de coeficientes e de análise de regressão linear baseado em parâmetros antropométricos de homens destreinados em força. Rev. Bras. Educ. Fís. Esporte, 23: 171-181. DOI: http://dx.doi.org/10.1590/S1807-55092009000200007

22. Miller A.E.J., J.D. MacDougall, M.A. Tarnopolsky, D.G. Sale (1993) Gender differences in strength and muscle fiber characteristics. Eur. J. Appl. Physiol. Occup. Physiol., 66: 254-262.

23. Munn J., R.D. Herbert, M.J. Hancock, S.C. Gandevia (2005) Resistance training for strength: effect of number of sets and contraction speed. Med Sci. Sports Exer., 37: 1622-1626.

24. Neils C.M., B.E. Udermann, G.A. Brice, J.B. Winchester, M.R. McGuigan (2005) Influence of contraction velocity in untrained individuals over initial early phase of resistance training. J. Strength Cond. Res., 19: 883-887. DOI: 10.1519/R-15794.1

25. Ramalho G.H.R.O., M.L. Mazini Filho, B.M. Rodrigues, G.R.O. Venturini, R.S. Salgueiro, R.L. Pace Júnior, D.G. Matos (2011) The 1RM testing for prediction of load in hypertrophy training and its relation with maximum number of repetitions. Braz. J. Biomotricity, 5: 168-174.

26. Sakamoto A., P. Sinclair (2006) Effect of movement velocity on the relationship between training load and number of repetitions of bench press. J. Strength Cond. Res., 20: 523-527. DOI: 10.1519/16794.1

27. Santiago F.L.S., G.A. Paz, M.F. Maia, P.S. Santos, A.T.L. Santos, V.P. Lima (2012) Strength of maximum repetitions and tension time on leg press after stactic elongation in extensor and flexor knee. Rev. Bras. Prescr. Fisiol. Exerc., 6: 3-9.

28. Shephard R.J. (1988) PAR-Q: Canadian home fitness test and exercise screening alternatives. Sports Med., 5: 185-195.

29. Spagnol A.R., O.C.M. Malheiro, R.C. Castoldi, D.G. Moret, R.G. Araújo, M. Papoti, R.C.T. Camargo, J.C.S. Camargo Filho (2012) Análise da plasticidade muscular de ratos submetidos a um protocolo de treinamento físico concorrente. Rev Bras Ciênc Mov., 20: 118-124. DOI: http://dx.doi.org/10.18511/rbcm.v20i3.3607

30. Tanimoto M., N. Ishii (2006) Effects of low-intensity resistance exercise with slow movement and tonic force generation on muscular function in young men. J. Appl. Physiol., 100: 1150-1157. DOI: 10.1152/ japplphysiol.00741.2005

31. Thomas J.R., J.K. Nelson, S.J. Silverman (2012) Métodos de pesquisa em atividade física. 6th ed. Rio de Janeiro: Artmed. 
32. Watanabe Y., M. Tanimoto, A. Ohgane, K. Sanada, M. Miyachi, N. Ishii (2013) Increased muscle size and strength from slow-movement, low-intensity resistance exercise and tonic force generation. J. Aging Phys. Act., 21: 71-84.

33. Wernbom M., J. Augustsson, R. Thomeé (2007) The influence of frequency, intensity and volume and mode of strength training on whole muscle cross-sectional area in humans. Sports Med., 37: 225-264.

34. Westphal M., R.R. Batista, A.R. Oliveira (2006) Relationship between body mass, lean body mass, cross sectional area and $1 \mathrm{RM}$ in women. Rev. Bras. Cineantropom. Desempenho Hum., 8: 52-57.

35. World Medical Association (1989) Declaration of Helsinki. Recommendation guiding physicians in biomedical research involving human subjects. Java. 227: 925-926.

\section{Received 27.07.2016 \\ Accepted 28.11.2016}

(C) University of Physical Education, Warsaw, Poland 